\title{
PLATAFORMAS DE DIFUSIÓN DE CONTENIDOS AUDIOVISUALES PARA TELEFONÍA MÓVIL: REALIDAD Y POTENCIALIDAD
}

\author{
Jorge Caldera-Serrano \\ Departamento de Información y Comunicación - Universidad de Extremadura \\ jcalser@unex.es \\ José-Antonio León-Moreno \\ Consultor TIC - Universidad de Extremadura \\ yo@jaleon.es
}

\section{Resumen}

\begin{abstract}
Se analiza la utilización de teléfonos móviles para el acceso a contenidos audiovisuales en directo como VoD. Para ello se realiza un recorrido por el desarrollo y evolución de la telefonía móvil y el uso por parte de la población, para destacar su gran implantación en el mercado mundial. De ahí se analiza la expansión de la presencia y uso del teléfono móvil como herramienta de acceso a contenidos audiovisuales, analizando finalmente las principales plataformas españolas con sus principales características técnicas. Se incluyen también potenciales líneas de trabajo y modelos de negocios que están surgiendo en el desarrollo del uso de la telefonía para el acceso a contenidos televisivos.
\end{abstract}

Palabras claves: Telefonía móvil; plataformas audiovisuales; difusión de contenidos; televisión red; cibertelespectadores

\section{INTRODUCCIÓN}

Marck Prensky (1946), precursor y defensor de las tecnologías del entretenimiento en el ámbito educativo, además de ser quién acuñó y difundió el concepto de "nativos" e "inmigrantes digitales" (2001), defendía en el programa de Televisión Española "Redes" (2010), presentado por el incombustible Eduardo Punset, que nuestros jóvenes habían nacido rodeado de "pantallas", que dichas "pantallas" les habían acompañado a lo largo de su vida, que les habían ayudado a formarse, a educarse, a entretenerse, en definitiva, que habían sido una pieza clave en el proceso educativo y madurativo del niño, de tal manera que ese acompañamiento de "pantallas" estaba preparando al niño para un entorno muy distinto al medio ambiente de sus padres, en el que las Tecnologías de la Información y la Comunicación no son una pieza fundamental del desarrollo de la persona sino que era la pieza sobre la que se sustentaba su formación, su entretenimiento, su adquisición de conocimiento, su adiestramiento, etc.; sí, las TIC y su estrecha relación con lo audiovisual están claramente desbancando a otros paradigmas anteriores en ámbitos tan diversos como la educación, el entretenimiento, la comunicación, etc. A los jóvenes que habían crecido en este ambiente, Prensky los denomina "nativos digitales".

Estas pantallas a las que se refiere Prensky son las de la computadora, la televisión, las pantallas de los videojuegos en sus diferentes versión (Wii, PSP, etc.), tablets, laptops, i-pad, así como los i-phone y los smartphones.

Pero existe una clara corriente integradora tecnológica, en la que todas las potencialidades de otras herramientas se conjugan en una única: el celular o teléfono móvil. En el mismo aparato se integra los elementos propios de un teléfono, su movilidad, herramientas para la conexión a la red, app para las interconexión con las redes sociales, grabación de audio, vídeo y fotografías, así como su almacenamiento y difusión, etc. y derivado de la mejora del ancho de banda, de la concurrencia en los servidores audiovisuales, y por supuesto una clava evolución en los equipos de telefonía móvil, la visualización de contenidos audiovisuales se está desarrollando y asentando a pasos agigantados. El crecimiento exponencial de usuarios consumidores de contenidos audiovisuales 
también se está viendo acompañada por la apuesta por la difusión de contenidos propios para teléfonos.

Dicha apuesta rompe con las cadenas del tiempo y espacio que provocaba la visualización de contenidos por televisión, y aunque la "televisión a la carta" en cierta manera ya había conseguido dicha evolución, ahora nos encontramos con la revolución de la ubicación del receptor, del ciberespectador, y de la potencialidad de consumo de contenidos con gran agilidad, ubicuidad e incluso de bilocación (conexión compartida de productos audiovisuales de forma simultanea).

Actualmente ya existen experiencias, aún en fase de desarrollo, en la cual se están poniendo a disposición del cibertelespectador galerías de contenidos sólo accesibles por medio de celulares, y su consumo así como su calidad, están siendo desarrollados de forma trepidante.

Este trabajo no sólo quiere analizar la situación actual de la difusión de estos contenidos por medio de plataformas específicas para teléfono móviles, sino que lo que se quiere es identificar aquellos elementos en los que existe un claro desarrollo y un claro potencial, e intentar vislumbrar -a la vista de los avances tecnológicos- cuáles son los retos que debe afrontar la telefonía móvil.

\section{OBJETIVOS Y METODOLOGÍA}

El objetivo del trabajo es la identificación, descripción y análisis tanto de las plataformas que difunden contenidos a teléfonos móviles cómo de los requerimientos de los dispositivos. Para lograr igualmente este objetivo será necesario la identificación y valoración de las app necesaria para el uso de estas plataformas así como los requerimientos mínimos de equipos y programas. También quisiera lograr el facilitar aquellas potencialidades y utilidades futuras que pudieran ser implementadas para el uso de esta tecnología para una mayor y mejor difusión y uso.

La metodología se fundamenta en una serie de etapas, no siempre secuenciales, para así poder lograr el objetivo del trabajo.

La primera fase, como en cualquier trabajo de investigación, es la búsqueda de información en bases de datos como repositorios de información científica, así como en repositorios $y$ otras fuentes referenciales. Igualmente útil es la consulta de revistas de difusión tecnológica y blogs que nos marcan la tendencia en consumo, investigación y desarrollo tecnológico en materia audiovisual y en telefonía.

La segunda fase ha analizado las principales plataformas de difusión de contenidos. Para ello se han identificado, y posteriormente, se han analizado para conocer contenidos, alcance y difusión.

En un tercer momento, se han analizado las Apps necesaria para la interconexión y comunicación entre la telefonía móvil y las plataformas de distribución de contenidos audiovisuales, así como las características básicas y mínimas de los celulares para visualizar la información.

Como cuarta fase, se han utilizado dichas plataforma como consumidor, pudiendo así detectar la realidad de estas plataformas.

Atendiendo al estado de la cuestión, la evolución tecnológica y la demanda de los usuarios, se identifican algunos elementos que pueden contar con interés en el desarrollo evolutivo de este tipo de consumo de contenidos audiovisuales, identificando los cambios en el modelo de negocio que plantea este método de difusión.

\section{TELEFONÍA MÓVIL: CRECIMIENTO EXPONENCIAL}

La Unión Internacional de Telecomunicación (UIT) presentó hace en 2013 su informe anual de "Medición de la sociedad de la información" (2013) en el que aborda, entre otros factores relacionados con el uso y penetración de las TIC, el rápido crecimiento y potencial de la telefonía móvil como elemento de comunicación cada vez con mayor importancia y uso.

Además de recomendar la lectura del documento completo o en su formato de resumen ejecutivo (UIT, 2013) la propia organización destaca en su UITNews los datos principales sobre la telefonía (UITNews, 2013).

Además de algunos de los datos que hemos extraído del estudio, cabe destacar incluso el titular aportado "Teléfonos móviles: ya son casi 7.000 millones itenemos casi todos un teléfono?". El estudio realiza una prospección que para finales del 2013 el número de teléfonos móviles estaría a la par con la población mundial, y que dicha evolución hará 
que en el año que 2014 el número de líneas de teléfono móvil sea superior al global de la población mundial.

Figura 1: Convergencia de las tendencias de los abonos móviles-celulares y de la población mundial

\section{Convergencia de las tendencias de los abonos móviles-celulares y de la población mundial}

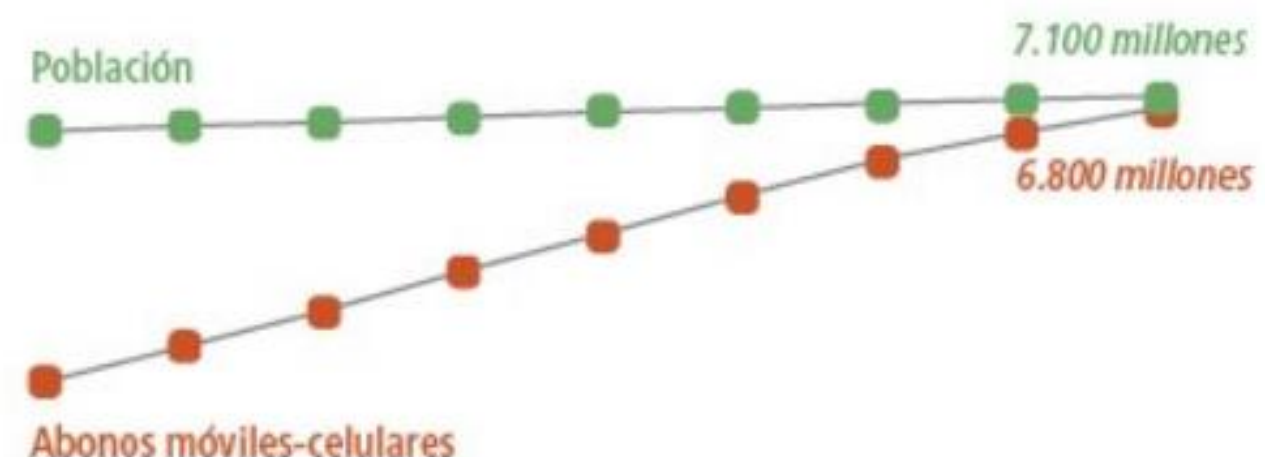

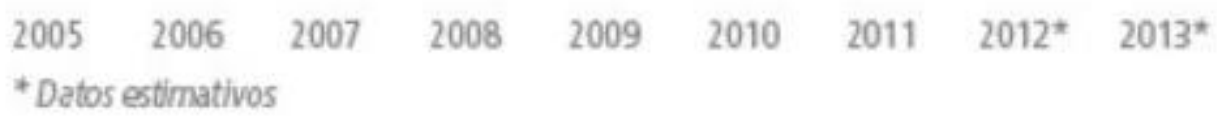

Fuente: ITU News ${ }^{1}$

No obstante, estos números no significan que todos los habitantes del planeta tengan teléfonos móviles. La GSMA (2013) en su informe sobre la "economía móvil" señala que el número de abonos móviles de carácter individual es de 3.400 millones (número nada desdeñable, evidentemente). Esto significa que estas personas cuentan con más de una línea de telefonía móvil (privado, laboral, etc.). Además, en muchas zonas del mundo se utiliza la opción de "prepago", por lo que una misma persona puede contar con un número significativo de tarjetas "Sim", con el fin de no pagar cuotas fijas. Mientras que en otros países la tasa de pre-pago es baja al contar sólo con contratos, siendo la penetración baja aunque el uso del móvil es mucho más estandarizada, como por ejemplo Japón o Corea.

Otra variable que hace que esta estadísticas haya de ser tenida en cuenta de forma relativa es, por un lado, que algunos operadores tardan mucho tiempo en dar de baja líneas inactivas, y la realidad de que en zonas en desarrollo una misma línea puede ser utilizada por más de una persona.

Es también importante destacar que el número de móviles con conexión de banda ancha, lo que sería especialmente interesante para el potencial del producto que se está analizando en este trabajo, es de 2.100 millones en el año 2013. En países desarrollados el uso del móvil con banda ancha se complementa con el uso de banda ancha fija, no así en países en desarrollo donde la banda ancha móvil sustituye al fijo.

Se entiende por la UIT banda ancha a aquellos abonos móviles con velocidades como mínimo de $256 \mathrm{kbit} / \mathrm{s}$, que garantizan el acceso a datos a través de Internet. Indicar que más de la mitad de los abonados a la telefonía celular están en la región de Asía y el Pacífico.

1 Disponible en: https://itunews.itu.int/Es/3780-Telefonos-moviles-ya-son-casi-7000-millones-brTenemos-casi-todos-un-telefono.note.aspx 
Figura 2: Abonos móviles-celulares en todo el mundo (para finales de 2013)

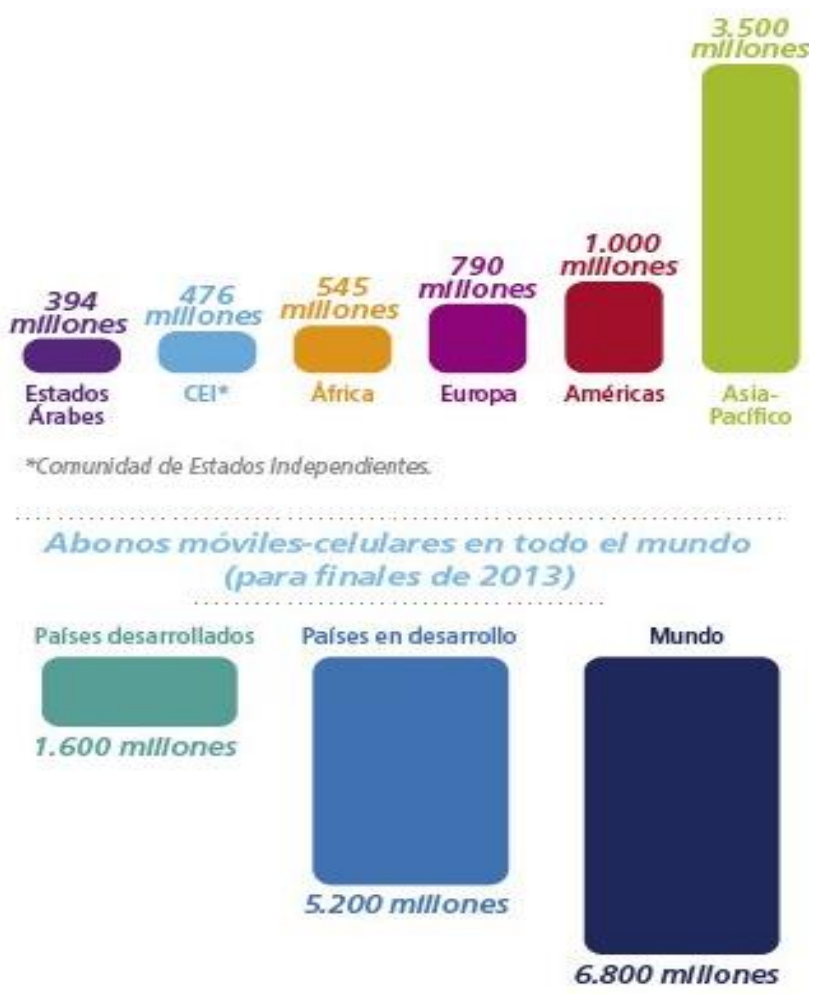

Fuente:ITU News ${ }^{2}$

Sobre España cabe destacar que en mes de agosto de 2014 la telefonía móvil contaba con más de 50 millones de abonos, siendo la tasa de penetración del 109,3\%, por lo tanto, se cuentan con más líneas que habitantes (Observatorio, 2014). Estos más de 50 millones de líneas corresponden a líneas de prepago, postpago y datacards. Las líneas asociadas a máquinas (M2M) se acerca a los tres millones (2,8 millones). Las líneas prepagos son aproximadamente 14,24 millones y las postpago 35,61 millones.

2 Disponible en: https://itunews.itu.int/Es/3780-Telefonos-moviles-ya-son-casi-7000-millones-brTenemos-casi-todos-un-telefono.note.aspx 


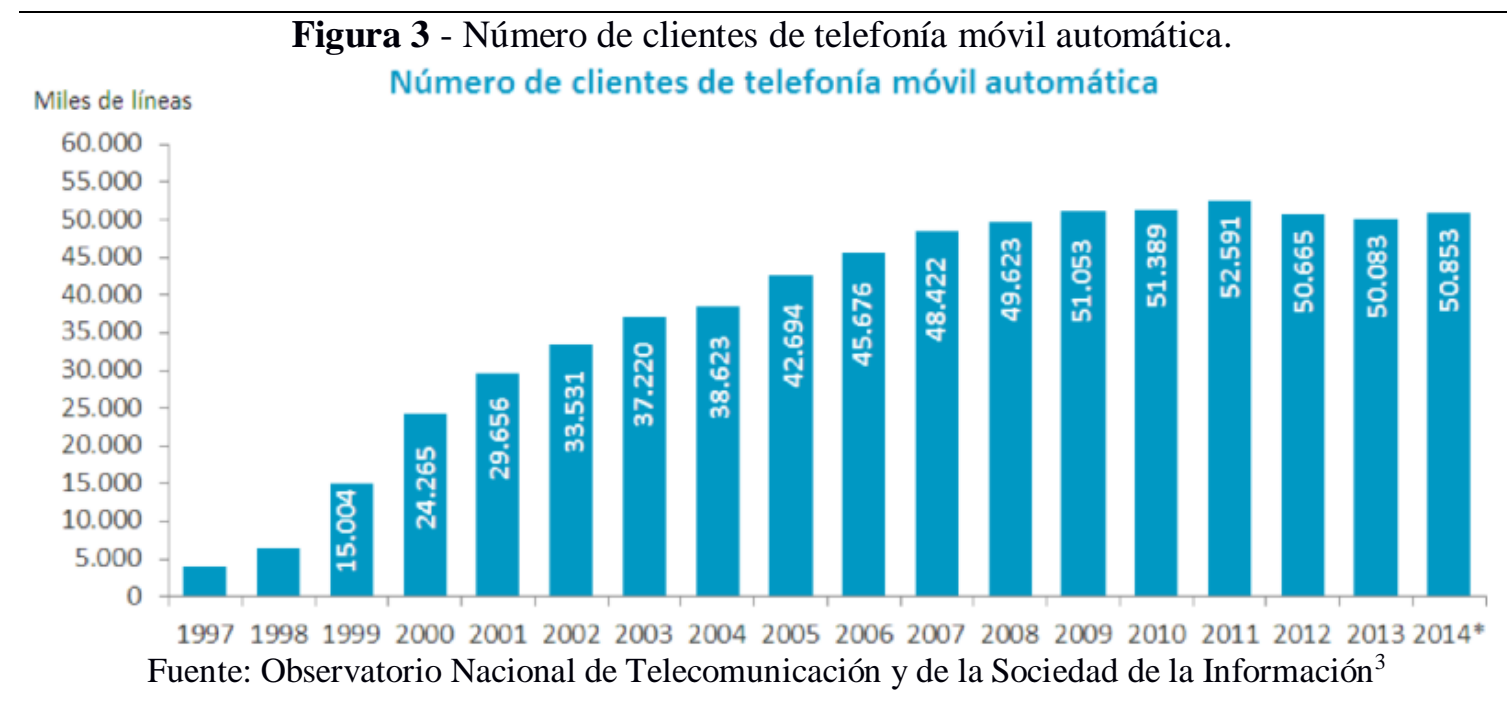

El INE (2014) ha presentado recientemente su encuesta sobre equipamiento y uso de tecnologías de información y comunicación en hogares, donde destaca que el $74,4 \%$ de los hogares tienen conexión a internet, aunque es destacable que existen más usuarios de internet $(76,2 \%)$ que de ordenadores $(73,3 \%)$, lo que supone según el estudio que un $77,1 \%$ de los internautas accedieron a internet por medio de la telefonía móvil.

En resumen, el teléfono móvil-celular cuenta con una amplia penetración en todo el mundo, situándose España en la parte alta de la tabla de entre los países que se conectan a internet por medio de los celulares. Sin lugar a dudas esto supone la mejor situación para poder acceder a contenidos audiovisual por medio de la telefonía móvil.

\section{TELEVISIÓN 2.0: POTENCIANDO LA INTERCONEXIÓN MÓVIL}

Existe una clara convergencia entre la televisión y las redes sociales. Desde las televisiones, cada vez más, se potencia la presencia y la interconexión por medio de Facebook y Twitter, fundamentalmente. Se plantea la interacción y comunicación no sólo con los medios televisivos sino entre aquellos que consumen, a la vez, un mismo producto audiovisual. Adelantado y Martí (2011) realizan un estudio sobre la situación del acceso a los contenidos audiovisuales por móviles.
Todas las cadenas de televisión, las importantes al menos, cuentan con departamentos de contenidos digitales en los que también se busca el captar a un nuevo espectador, el nativo digital, que tiene unos hábitos distintos de consumo televisivo y de acceso al entretenimiento.

Tal y como señala Lorente (2011) existen diversas definiciones del concepto de Televisión Social con orientaciones muy diversas, centrando su apreciación desde los componentes tecnológicos y otros en los elemento más sociales. Se destacan las definiciones de Duchenaut (2008), Harboe (2008), Metcalf (2008), Chorianopoulos y Lekakos (2008), Hayes (2010), Mitchell (2010), Vinader y Abuin (2010), Montpetit (2010), Kastelein (2011), entre otros.

Por lo tanto, la televisión social podría definirse como la nueva tecnología de televisión interactiva social, donde el espectador puede participar de diferentes maneras en tiempo real, a través de chat de audio y texto, videoconferencias, como otras potenciales maneras de intercomunicación, utilizando para ello especialmente las redes sociales (Facebook, Twitter, etc.) a través de dispositivos auxiliares, tales como computadoras, tablets, i-pad y teléfonos celulares.

La Televisión Social ha tomado mucho de los elementos de plataformas de difusión de contenidos audiovisuales que se ha demostrado muy interesante para un perfil concreto de usuarios. YouTube, por ejemplo,

\footnotetext{
${ }^{3}$ Disponible en: http://www.ontsi.red.es/ontsi/node/1071
} 
ha facilitado la opción de interactuar con el propio contenido audiovisual. La mejora de la Televisión Social es la potencialidad de poder intercomunicarse de forma sincrónica un número importante de usuarios con intereses comunes (en plataformas como las indicadas con anterioridad, la relación entre espectadores es asíncrona).

Las televisiones está potenciando la interconexión por medio de elementos externo a la propia televisión con el fin de fidelizar a los nativos digitales con nuevos métodos de consumo, de tal manera que de forma paralela a la Televisión Social los medios están desarrollando estrategias para facilitar información directamente para celulares y para acceder a contenidos por medio de este dispositivo.

A continuación exponemos el gráfico de Morales (2011) para analizar como hace ya un par de años se estaba desarrollando estrategias más allá de la Televisión Social, vislumbrando posiblemente la potencialidad de contar en una misma herramienta la facilidad de acceder a las redes sociales y al contenido audiovisual: teléfonos móviles.

Figura 4: Resumen y características de productos de ficción para móviles-celulares Fuente: Morales (2011)

\begin{tabular}{|c|c|c|c|c|c|c|}
\hline Programa & Canal & Productora & Segmento & No. capítulos & $\begin{array}{l}\text { Duración } \\
\text { por episodio }\end{array}$ & $\begin{array}{l}\text { Forma de } \\
\text { descarga }\end{array}$ \\
\hline Supervillanos & Amena & Globomedia & Juvenil & 40 & $3 \mathrm{~min}$. & directa \\
\hline Intrigo & Vodafone & Fox & Juvenil & 24 & $5 \mathrm{~min}$. & directa \\
\hline A Pera picada & TV3 & CCRTV Interactiva & Juvenil & 13 & $2 \mathrm{~min}$. & Via web \\
\hline 13 anys i 1 dia & TV3 & $\begin{array}{l}\text { Mediápolis- } \\
\text { La Productora }\end{array}$ & Familiar & 14 & $24 \mathrm{~min}$ & Via web \\
\hline The imp & TV3 & Screen 21/ TVC & Infantil & 49 & $1.15 \mathrm{~min}$ & Via web \\
\hline Angus \& Cheryl & TV3 & BRB int. & Infantil & 104 & $1,5 \mathrm{~min}$. & Via web \\
\hline Nueve meses & XD & Maragda Mediática & Juvenil & 18 & $3.5 \mathrm{~min}$. & $X D$ \\
\hline
\end{tabular}

El uso de la Televisión Social en España es evidente. Benítez (2013) aporta datos contundentes. Según el estudio de Social TV de MPG junto con Salvetti\%Llombart, el 74\% de la población realiza multitasking mientras visualiza televisión, y de éstos la mitad están comentando contenidos televisivos (más de siete millones). Aplicaciones propias para interactuar por medio de las redes sociales y con información variada sobre la programación de la cadena son realidad. Algunas aplicaciones son Intonow, Viggle, Zeebox o Tockit para España.

\section{PRINCIPALES EXPERIENCIAS DE VOD PARA DISPOSITIVOS MÓVILES Y SUS CARACTERÍSTICAS}

En España contamos con intentos empresariales para facilitar Video bajo Demanda, lo cual no deja de ser un atrevimiento en un país que cuenta con un índice alto de descargas de contenidos no de pagos. Estos intentos son Wuaki, Yomvi, Filmin y Netflix, los que actualmente copan un mercado incipiente y poco asentado.

Wuaki cuenta con aproximadamente unos 1.850.000 suscriptores a nivel global y casi 1.400.000 en España y más de 125.000 en Reino Unido donde también opera tras su compra por la empresa japonesa Rakuten en 2012. Su modelo de negocio cuenta con tarifa plana de acceso $\mathrm{y}$ alquiler puntual.

Yomvi, de Canal+, comenzó el 2014 con cerca de 500.000 clientes. No tiene un coste adicional para los clientes de Canal+, por lo que es un complemento perfecto de su emisión tradicional. A día de hoy, son 
escasos los que acceden a sus contenidos fuera de los abonados de Canalt.

Filmin, fundada en 2006 por las productoras de cine Alta Films, Avalon Distribución y Cameo, cuenta con una tarifa plana mensual para acceder a cierta parte del catálogo, poniendo a disposición de los usuarios las películas de estreno por medio de alquiler. Filmin, además, ha llegado a acuerdos con El Corte Inglés para ofrecer dichos contenidos desde su web.

Estas plataformas están subiendo de forma constante, y parecen imponerse en estos momentos, aunque existen otras como Cineclick, Filmotech, Nubebox o Vimeo On Demand.

También destacamos Apple, que a través de iTunes ofrece la opción de compra y de alquiler de contenidos, contando con una facturación muy importante por la descarga y visionado de contenidos audiovisuales.

Volvemos a señalar que este mercado es incipiente, y que aún está por definir cómo serán utilizadas y qué valor darán los usuarios a sus contenidos. El pago por visualización en vídeo bajo demanda es cuestión de hábitos de consumo que, en España, aún no se tiene de manera arraigada.

Incluso se llega a señalar lo complicado de mantener de manera local estos modelos de negocio, vislumbrando una edad de fortalecimiento con la llegada de Netflix y Amazon, grandes distribuidoras internacionales de contenidos audiovisuales bajo demanda (Novoa, 2014).

Las compañías como Hulu, Yomvi, Netflix, funcionan de manera similar y aunque actualmente emiten en formato UltraHD, dependiendo del ancho de banda del proveedor y del dispositivo de recepción, la calidad no suele tener una resolución inferior a $720 \mathrm{p}$, es decir, el mínimo actual con la que se emite la televisión convencional en España a través de los canales de Televisión Digital Terrestre.
Todas estas empresas funcionan de manera similar siendo productos del denominado "Cloud Computing v1.0" lo que demuestra los enormes beneficios y también sus problemas.

Cloud Computing v1.0 es estrictamente una particularidad de Amazon Web Services (AWS), que fue el primero en ofertarlo cuando ningún otro proveedor tuvo las características centrales necesarias para crear aplicaciones comparables, actualmente Google puede competir en igualdad de condiciones.

Netflix eligió Amazon por sus múltiples zonas de disponibilidad y EBS con instantáneas y restauraciones rápidas. Tiene sentido, pues, que Netflix haya adoptado AWS; vio enormes beneficios de poder implementar y escalar su servicio utilizando las interfaces y arquitecturas que fueron posibles cuando se lanzó AWS.

EL modo de uso es que cuando alguien le da 'play' a un vídeo, la nube lo guía a un Content Delivery Network (CDN), desde donde se envía el vídeo, ya que la CDN está diseñada para tener los vídeos lo más cerca posible. Muchos proveedores de servicios tienen aplicaciones de Netflix dentro de sus centros de datos ya que permite el almacenaje de películas y permite así el balanceo de carga, no exigiendo un overcloakin o saturación de determinados servidores, así como disponer de conexiones directas en muchos de los grandes centros de intercambios de la red. A modo de servers o mirror donde el usuario puede ver un contenido en streaming.

Entrando en detalle técnico dentro de AWS, utilizan instancias de Windows para la codificación de vídeo. Y para el almacenamiento de vídeos, por ejemplo, usan decenas de miles de instancias de EC2 y petabytes de almacenamiento en S3.

Para tener una concepción de la magnitud del uso que hacen, podemos reseñar que con una única instancia podríamos dar soporte a varios sites 
dinámicos, alojar servicio de e-commerce, gestionar varías bases de datos. etc.

Utilizan Hadoop y Amazon EMR para su gestión, pues necesitan gestionar un terabyte al día de datos.

En cuanto a código, este tipo de plataformas suele estar basado en Java. Aprovechando bastantes herramientas Open Source y se han encontrado con que muchas herramientas de gestión de centros de datos no les valían, ya que ese código estaba orientado a centro de datos tradicional y en su transición a un sistema basado en la nube de Amazon prefirieron reescribir todo el código de cero. Pasaron a utilizar un sistema NoSQL como SimpleDB, memcached y protocolos más robustos y flexibles como Apache Avro.

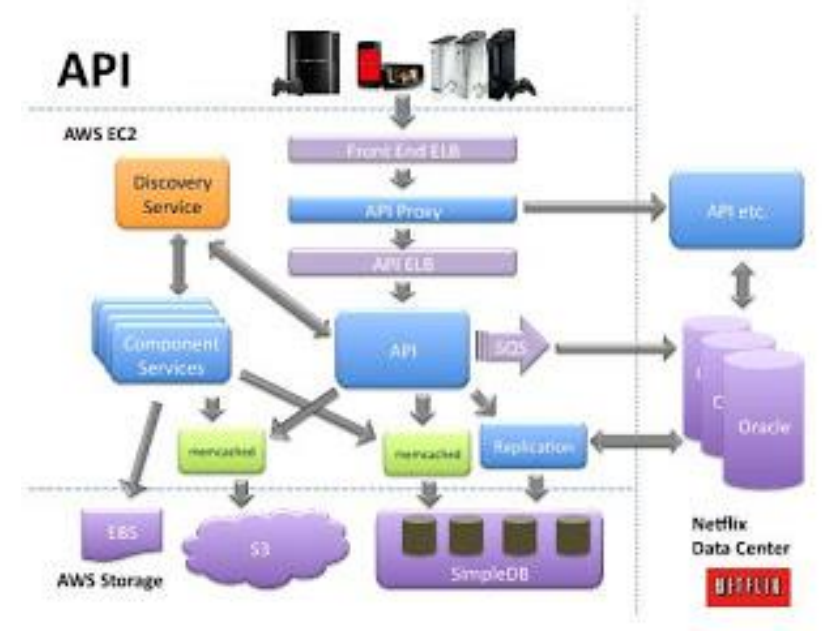

Utilizan Eclipse, Jira, Hudson, Ivy y Artifactory. La build se encarga de todo. Desde el WAR al RPM a la imagen de Amazon. Los servidores corren en sistema operativo CentOS, y utilizan Apache, Tomcat y OpenJDK.

Uno de los problemas de los desarrolladores es la colisión entre instancias cuando están realizando tests. Para ello crearon pequeños hacks de forma que se crean instancias por desarrollador y no existe esa colisión al desplegar.

Tienen su propia gestión de pools de threads ya que se preocupan bastante por el tamaño de los objetos y por cómo se pasan los objetos entre servicios: por referencia dentro del mismo servicio y por valor entre servicios. Los diferentes servicios instrumentan todas las llamadas. Utilizan diferentes objetos "fake" para que los desarrolladores puedan comenzar a trabajar lo más pronto posible, etc.

La compañía ha sido pionera en el desarrollo de aplicaciones en la nube, de hecho tiene aproximadamente unos 700 ingenieros en plantilla que constantemente están creando diferentes programas para sacar el mayor provecho de la nube posible, realizando microcortes, pruebas de estrés, caída completa de la infraestructura, etc. para ello han diseñado programas como Caos Monkey y Caos Kong para simular esas incidencias y que han sido adoptadas por compañías como Intel o eBay o incluso el equipo de campaña de del Presidente estadounidense Obama, ya que la filosofía de trabajo de estas compañías en la nube es por medio de proyectos Open Source alojados en github.com/netflix y donde depositan el código fuente en formato abierto, para que todos puedan en primer lugar aprovecharlo y en segunda medida realizar aportaciones o commit.

Cabe destacar que al ser arquitecturas tan complejas, hay que asumir que muchas de sus necesidades no las puede cubrir Amazon Web Services, por lo que deben implementar sus propios servicios de infraestructura (Balanceo en middle-tier, encriptación y Caching), así como desplegar en sus propios Centros de Datos el procesamiento de pagos online. El streaming principal lo delegan a CDNs de Akamai y el pago a través de servicios ya consolidados como paypal o similares. 
El servicio que aporta en un futuro que según estimaciones sería finales de este año, principios del siguiente sería la emisión de eventos relevantes deportivos tipo olimpiadas, superbowl, y películas filmados en $4 \mathrm{k}$ lo que hará que la calidad de la emisión sea de una enorme resolución.

Se han generado a partir de esta realidad app para smarthphones así como para tablets, dando la posibilidad y potenciando el acceso a canales de televisión de diferentes países por medio de la transmisión en streaming.

Uno de los principales problemas es que la visualización mediante streaming provoca un elevado tráfico de datos, por lo se ha de ser consciente que podrán acceder a estos contenidos quién tenga realmente una tarifa plana de datos (o use conexión wi-fi).

Desde alguna de estas aplicaciones puede comentar por medio de Facebook y Twitter lo que se está visualizando, y todo desde el teléfono móvil.

Existen en el ámbito español app para acceder a canales oficiales, por ejemplo +tve (Televisión Española), +24 (de Canal 24 horas de Televisión Española), Clan (canal de programación infantil de Televisión Española), Atresplayer (acceso a contenidos de Antena 3 TV, La Sexta, Neox y Xplora), Mitele (acceso a contenidos de Tele 5 y Cuatro), Eurosport Player (con el acceso a los contenidos de Eurosport y Eurosport 2), TV3 (app para el acceso a los contenidos de la Televisión de Catalunya), Yombi (Canale de deportes de Canal+), Movistar TV (con el acceso a un amplio catálogo de películas y series), etc. Y siguen existiendo, cada vez más, las app para poder acceder a los contenidos audiovisuales de diferentes plataformas.

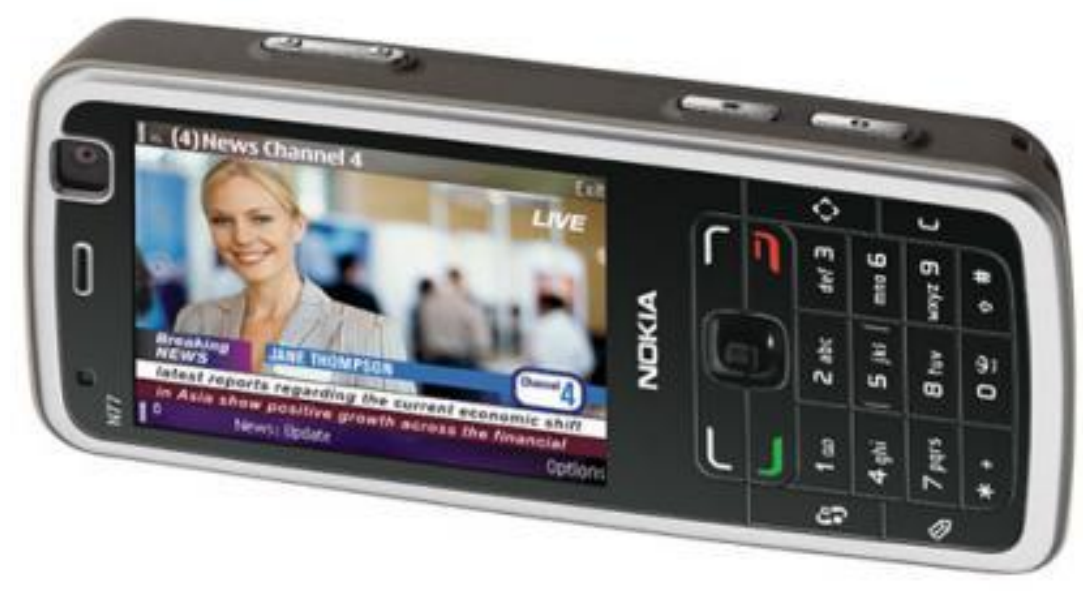

Fuente: http://universocelular.com/2008/05/19/tv-gratis-por-internet-desde-el-celular/

\section{CONCLUSIONES}

Es complicado el incidir en cuál será la tendencia en el uso y consumo de tecnología. Son muchos autores y experto quienes opinan que el teléfono móvil, por su tamaño y versatilidad, será el dispositivo en el cuál se aglutinen el mayor número de utilidades y será el más extendido y utilizado por los usuarios de tecnologías. Así es ya, sin lugar a dudas. Pero además, las grandes empresas de comunicación están realizando grandes avances y esfuerzos en ofrecer cada vez mejores equipos y también en facilitar mayores recursos a los cuales poder acceder.

Un único dispositivo en el que se pueda conjugar todas los requerimiento de los "nativos digitales", y parece que hoy el teléfono celular es la apuesta más segura.

E incluso se está apostando para el acceso a contenidos audiovisuales. Es cierto que en España aún no tiene una penetración comparada con la de otros países, debido seguramente a la tendencia al consumo ilegal de contenidos y a la falta de hábito del pago por visión, no obstante los números van en 
aumento, y la legislación intentan ayudar a la industria audiovisual por medio de legislación que dificultad el acceso a contenidos ilegales.

Actualmente ya contamos con plataformas que difunden contenidos en red para uso del teléfono móvil, en exclusividad, o que facilitan al acceso a estos dispositivos, además de a otros.

Por lo tanto, lo que es necesario un desarrollo de la industria y una mejora en lo referente a la tecnología y al ancho de banda (realidades tecnológicamente superadas en estos momentos)

Es complicado estimar cuál es el futuro de esta tecnología y la difusión de contenidos por medio de estas plataformas. Parece vital el mejoramiento el ancho de banda y el aumento de la cocurrencia en estas plataformas.

Desde el punto de vista tecnológico, parece que las sinergias con las Realidad Aumentada
(Caldera, 2014) ya sea utilizando otros periféricos (gafas, tablets, etc.) o utilizando el propio celular va a ir siendo una realidad, al igual que el desarrollo de redes sociales y/o app para fragmentar aún más la audiencia y el visualizado conjunto.

Las opciones de multipantalla en el mismo dispositivo con el fin de poder ver varias productos de la misma plataforma de difusión, e incluso de distintas, es igualmente un requerimiento en el cuál se están realizando esfuerzos de desarrollo.

Por lo tanto, la evolución tecnológica derivado de las múltiple compañías desarrolladoras hace esta carrera trepidante y veloz. Parece que vamos encaminados a la supremacía del teléfono móvil sobre otros periféricos a la hora del acceso a contenidos, derivado por su facilidad de uso y transporte.

\section{PLATFORMS DISSEMINATION OF AUDIOVISUAL CONTENT FOR MOBILE PHONES: REALITY AND POTENTIAL}

\section{Abstract}

It analyzed the use of mobile phone for access to audiovisual content live and VoD. To do a tour is done by the development and evolution of mobile phones and use by the population, to highlight its large presence in the global market. Hence, the expansion of the presence and use of mobile phones as a tool for access to audiovisual content is analyzed, finally analyzing the main Spanish platforms with its main technical characteristics. Potential lines of work and business models that are emerging in developing the use of the telephone to access television content are also included.

Keywords: Mobile phones; audiovisual platforms; dissemination of content; television network; cyberviewers

\section{REFERÊNCIAS}

ADELANTADO, Eulalia; MARTÍ, José. Contenidos audiovisuales y televisivos para dispositivos móviles: una aproximación al mercado español. AdComunica: Revista de Estrategias, Tendencias e Innovación en Comunicación, n.1, p. 99-113, 2011.

BENÍTEZ, Javier. La televisión social, ¿moda o realidad? 2013. Disponível em: http://www.territoriocreativo.es/etc/2013/05/1 a-television-social-\%C2\%BFmoda-orealidad.html
CALDERA-SERRANO, Jorge. Realidad aumentada en televisión y propuesta de aplicación en los sistemas de gestión documental. El Profesional de la Información, v. 23, n. 6, p. 643-650, 2014.

CHORIANOPOULOS, K.; LEKAKOS, G. Introduction to Social TV: enhancing the Share Experience with interactive TV. International Journal of Human-Computer Interaction, v. 24, n. 2, p. 113-120, 2008.

DUCHENEAUT, N.; Moore, R.J.; OEHLBERG, L., THORNTON, J.D.; NICKELL, E. Social TV: designing for distributed, sociable television viewing. 
International Journal of Human-Computer Interaction, v. 24, n. 2, p. 136-154, 2008.

HARBOE, G; MASSEY, N. et al. The uses of social television. Computer in Entertainment, v. 6, n. 1, p. 1-5, .2008.

HAYES, G. Social: the second coming of TV. Heralded by Moki TV? 2010. Disponível em: http:www.personalizemedia.com/social-thesecond-coming-of-tv-heralded-by-moki-tv/

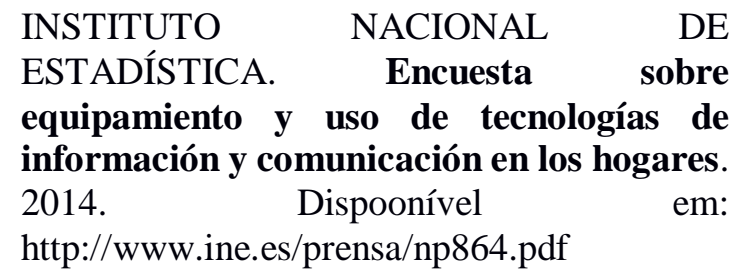

ITU News. Disponível em:

https://itunews.itu.int/Es/3780-Telefonosmoviles-ya-son-casi-7000-millones-br-

Tenemos-casi-todos-un-telefono.note.aspx

KASTELEIN, M.). Social TV: Blue Ocean Strategy? The Channel Magazine (The Asociation for International Broadcasting), v. 1, n.1, p. 21-23, 2011.

LORENTE CANO, M. Social TV en España: concepto, desarrollo e implicaciones. Cuadernos de Gestión de Información, p. 55-64, 2011.

METCALF, C.; HARBOE, G.; TULIO, J.; et al. Eamining presence and lighweight messaging in a social television experience. Journal ACM Transactions on Multimedia Computing, Communications and Applications, v. 4, n.4, p.1-16, 2008;

MITCHELL, K; JONES, A.; ISHMAEL, J; RACE, N.J.P. Social TV: toward content navigation using social awareness. Quality, p.28-291, 2010.

MONTPETIT, M.J. Relying on relationships to rebuild TV audiences Social TV. MIT Technology Review, v. 113, n.3, p. 55-56, 2010.

MORALES MORANTE, Luis (2011) Evolución tecnológica, estado actual y perspectivas. La producción de ficción para telefonía móvil. Revista Telos (Cuaderos de Comunicación e Innovación), p. 1-7, abr.-jun. $2011 . \quad$ Disponível em: http://telos.fundaciontelefonica.com/urldirect/pdfgenerator?tipoContenido=articuloTelos\&idCo $\underline{\text { ntenido }=2011050914020001 \& \text { idioma }=\mathrm{es}}$

NOVOA, Jaime. ¿Qué ha sido de los grandes proyectos de vídeo bajo demanda en España? 2014. Disponível em: http://www.genbeta.com/web/que-ha-sido-delos-grandes-proyectos-de-video-bajodemanda-enespana?utm_source=feedburner\&utm_mediu $\underline{\mathrm{m}=\text { feed\&utm_campaign }=\text { Feed } \% 3 \mathrm{~A}+\text { genbeta }}$ $+\% 28 \mathrm{Genbeta} \% 29$

OBSERVATORIO NACIONAL DE LAS TELECOMUNICACIONES Y DE LA SOCIEDAD DE LA INFORMACIÓN. Evolución del número de clientes de telefonía móvil en España. 2014. Disponível em: http://www.ontsi.red.es/ontsi/node/1071

PRENSKY, Marc. "Digital Natives, Digital Immigrants Part 1", v. 9, n. 5, p.1 - 6

REDES - PROGRAMA DE TELEVISIÓN"No me molestas mamá, estoy aprendiendo". Emitido por TVE el 5/12/2010 y 24/07/2011. Disponível em:http://www.rtve.es/alacarta/videos/redes/r edes-20-no-molestes-mama-estoyaprendiendo/953666/

UNIÓN INTERNACIONAL DE TELECOMUNICACIONES. Medición de la Sociedad de la Información: resumen ejecutivo. 2013. Disponível em: http://www.itu.int/en/ITU-

D/Statistics/Documents/publications/mis2013 /MIS2013-exec-sum S.pdf

VINADER Segura, R.; ABUÍN VENCES, N. Las estrategias digitales de los operadores de televisión: nuevos modelos de negocio e interacción en la era digital. In: Actas II CONGRESO INTERNACIONAL LATINA DE COMUNICACIÓN SOCIAL, 2., Laguna, 2010. Actas... Laguna: Universidad de la Laguna. Disponível em: Http://www.revistalatinacs.org/10SLCS/actas _2010/40Abuin333.pdf 


\section{Agradecimientos}

Este trabajo ha sido financiado por la Junta de Extremadura (Consejería de Educación, Ciencia y Tecnología) y el Fondo Social Europeo dentro del plan de apoyo a las actuaciones de los Grupos de Investigación inscritos en el catálogo de la Junta de Extremadura. GR10019. 